\title{
Des pièces de monnaie au cadenas
}

Un spectre des dépôts contemporains

From Coins to Locks : A spectrum of contemporary deposits

\section{Ceri Houlbrook}

\section{(2) OpenEdition}

Journals

Édition électronique

URL : https://journals.openedition.org/tc/9988

DOI : $10.4000 /$ tc. 9988

ISSN : 1952-420X

Éditeur

Éditions de l'EHESS

\section{Édition imprimée}

Date de publication : 30 octobre 2018

Pagination : 240-259

ISBN : 2-7132-2751-6

ISSN : 0248-6016

Référence électronique

Ceri Houlbrook, «Des pièces de monnaie au cadenas », Techniques \& Culture [En ligne], 70 | 2018, mis en ligne le 06 décembre 2020, consulté le 29 septembre 2022. URL : http://journals.openedition.org/ tc/9988; DOl : https://doi.org/10.4000/tc.9988 


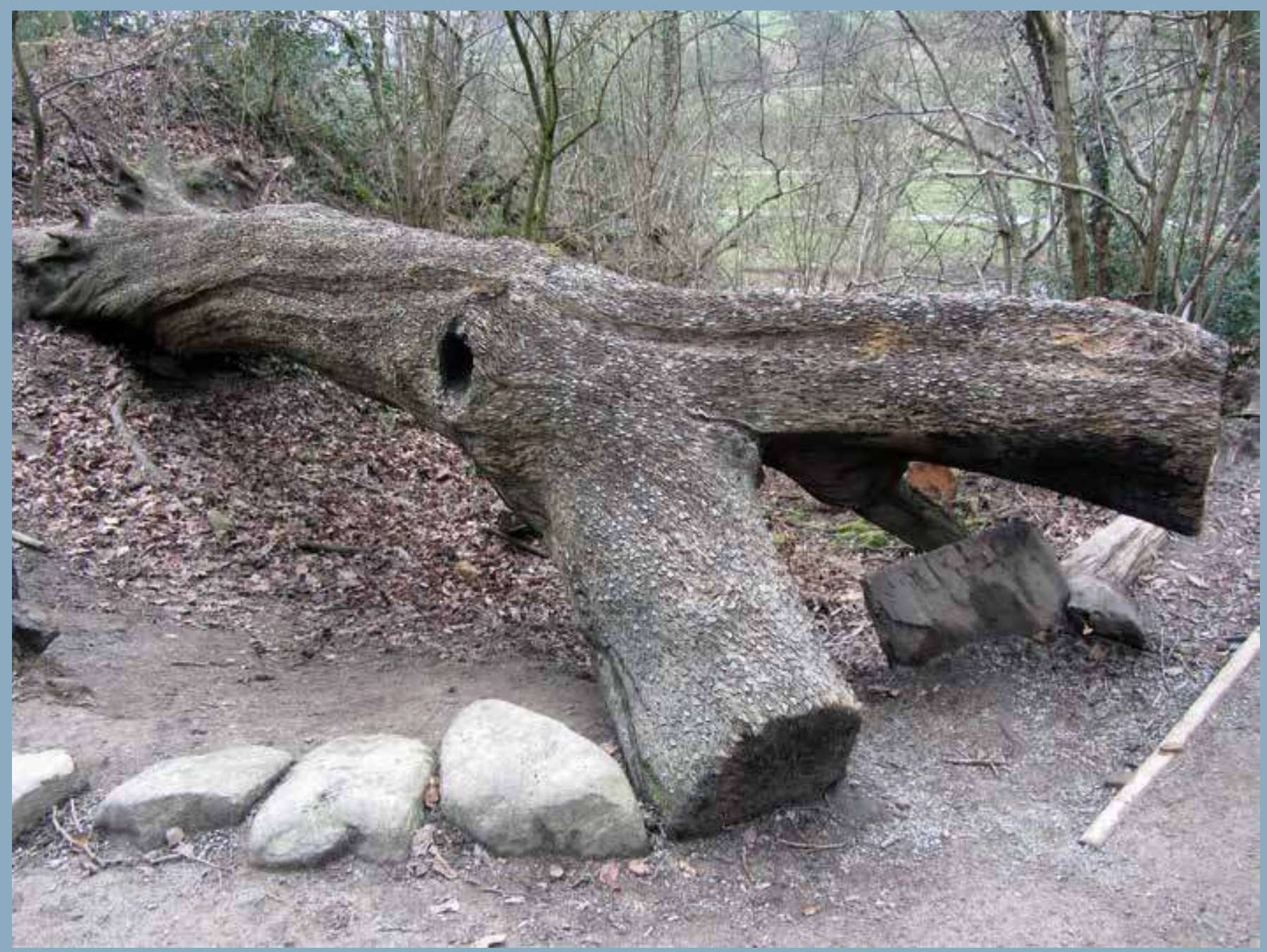




\section{Des pièces de monnaie au cadenas}

\section{Un spectre des dépôts contemporains}

On aurait tord de penser que les dépôts rituels n’appartiennent qu'au passé. En réalité, on pourrait même dire que cette pratique est aujourd'hui plus répandue, avec l'émergence un peu partout dans le monde de ce que le folkloriste Jack Santino (2004) désigne comme des «agencements populaires» (folk assemblages). Depuis le mémorial de bord de route et le cairn de montagne jusqu'à la fontaine à vœux, en passant par l'arbre à chaussures, nos paysages sont parsemés de dépôts collectifs. Lynne McNeill, elle aussi folkloriste, considère ces accumulations comme des «créations collaboratives en série», qui sont symptomatiques d'un état de la société actuelle. C'est en réaction à la nature toujours plus intangible et numérique des rapports humains et au manque de cohésion sociale qu'on apporterait une contribution physique à une œuvre ou à un lieu commun (McNeill 2007). Comme le remarque Cathy Preston, spécialiste des mythologies contemporaines, il s'agit de:

«former de manière cumulative un objet qui évoque une communauté imaginaire: une communauté constituée de différents individus, pour la plupart anonymes, qui ont répondu par la pareille à l'action de la personne précédente et qui envisagent souvent leur réponse comme les reliant à un groupe de personnes qui, bien qu'invisibles à leurs yeux, sont rendues visibles par ce qu'elles ont laissé derrière elles.» (Preston 2007)

J'ai montré dans une étude précédente que les agencements populaires contemporains sont, malgré leur succès (ou peut-être justement du fait de ce dernier), le plus souvent fortuits et improvisés (Houlbrook 2015 et 2018b). Cette caractéristique se manifeste, du moins dans les îles britanniques (la région qui fait pour l'essentiel l'objet de cet article), dans la nature même des dépôts qui sont généralement de faible valeur économique. Ainsi, les pièces jetées dans les fontaines sont des pièces d'un penny plutôt que d'une livre; les chaussures suspendues aux branches des arbres sont de vieilles baskets plutôt que des Jimmy Choo. On pourrait dire que 
ce fait reflète le faible niveau de croyance attaché aujourd'hui à ces rituels d'échange. En effet, si ces rituels sont pratiqués, c'est semble-t-il plus pour le sentiment de collaborer à une «communauté imaginaire» qu'au nom d'une croyance en leur efficacité.

J'ai montré ailleurs que la commodité est également un facteur de poids dans le choix des objets déposés (Houlbrook 2018a). C'est aussi ce que conclut Tristan Hulse dans son étude de la chapelle de Saint-Trillo à Llandrillo-yn-Rhos au Pays de Galles, qui est réputée être la plus petite chapelle du Royaume-Uni, construite sur un site consacré à saint Trillo depuis le vie siècle. En se rendant sur ce site dans les années quatre-vingt-dix, Hulse y a observé un agencement important d'objets contemporains constitué principalement de prières écrites à la main. Il a remarqué que la majorité d'entre elles étaient inscrites sur des bouts de papier trouvés au fond d'une poche ou d'un sac à main: enveloppes, tickets de caisse, pages arrachées à des cahiers ou tickets de transport en commun (Hulse 1995) - autant de caractéristiques indiquant la spontanéité de ces dépôts. Si les visiteurs déposent des objets qu'ils ont sous la main (par exemple des pièces dans une fontaine) ou qu'ils ont trouvés sur place (les pierres sur les cairns), c'est parce qu'ils ne sont pas allés voir un site particulier avec l'intention d'y apporter une contribution. De fait, il semble que, dans la majorité des cas, ce ne soit pas de manière délibérée que l'on rende visite à un dépôt : les visiteurs tombent dessus par hasard et ressentent ensuite le désir d'y contribuer (Houlbrook 2016). Pour reprendre une nouvelle fois Jack Santino, ces assemblages constituent des «invitations à participer» (2004).

Cependant, tous les dépôts contemporains ne sont pas effectués de manière spontanée. Certains sont de toute évidence planifiés: on achète ou on confectionne un objet avant de se rendre sur le lieu voulu, dans le but explicite de déposer cet objet sur place. Ces pratiques qui, même en l'absence de croyance, reflètent un plus fort investissement émotionnel dans le rituel, indiquent que tous les agencements populaires contemporains ne sont pas fortuits et improvisés. Pour examiner cette apparente dichotomie entre dépôts spontanés et dépôts planifiés, notre article s'appuiera sur deux formes d'agencements contemporains: l'arbre à pièces et le pont auquel sont attachés des cadenas d'amour. Au-delà de leur modernité, ces deux formes ont en commun leur caractère non institutionnel et non religieux (dans la plupart des cas), ainsi que leur lien au tourisme, puisque ces versements sont effectués en premier lieu par des touristes. Par ailleurs, comme nous le verrons, la pièce de monnaie et le cadenas d'amour sont tous deux apparentés à l'ex-voto dans la mesure où ces objets sont abandonnés dans le contexte d'un vœu. 


\section{Une brève présentation de l'arbre à pièces}

Cet article n'a pas pour objet de livrer une étude détaillée des arbres à pièces. Il s'agit plutôt de les utiliser comme support d'une réflexion théorique sur la pièce de monnaie en tant que dépôt improvisé: aussi ne présenterons-nous qu'un aperçu de cette pratique ${ }^{1}$.

Les arbres à pièces (coin trees) sont, comme leur nom l'indique, des arbres, ou le plus souvent, des souches dans l'écorce desquels sont incrustées verticalement des pièces de monnaies. On recense à l'heure actuelle 40 sites contenant plus de 200 arbres à pièces, répartis à travers toute l'Angleterre, le Pays de Galles, l'Écosse, l'Irlande du Nord et la République d'Irlande. Ces sites se trouvent souvent dans des zones rurales accessibles, en lisière de zones boisées appréciées du public ou de chemins de promenade sur les berges de cours d'eau. Sur les 40 sites, 37 sont encore «actifs», c'est-à-dire que des personnes participent aujourd'hui encore à la coutume consistant à insérer des pièces dans ces arbres. Une étude de terrain réalisée en 2011-2013 a consisté à cataloguer toutes les pièces déposées dans/sur des arbres à pièces: celles-ci sont en tout au nombre de 165360 .

Ce travail de terrain a permis de collecter un ensemble de données empiriques: les dates, la nationalité et l'état des pièces; le type d'arbres; leur situation dans le paysage. Des données d'ordre ethnographique ont aussi été recueillies afin de comprendre comment et pourquoi des personnes participent à cette coutume. Outre l'observation de participants sur chaque site, j'ai conduit des entretiens avec plus de 200 personnes pour constituer un échantillon représentatif. La majorité d'entre eux (82\%) étaient des touristes nationaux, avec une faible proportion de touristes internationaux $(10 \%)$ et une part plus faible de résidents locaux $(8 \%)$ définis comme vivant à moins de 30 kilomètres de l'arbre à vœux. Aucune surreprésentation d'un genre ou d'un groupe d'âge n'a pu être détectée. Parmi les participants, on comptait des couples de tous âges, des groupes d'amis, de nombreux groupes familiaux accompagnés de jeunes enfants, des habitants de la région venus promener leur chien. Toutes ces personnes avaient inséré une pièce dans un arbre à vœux ou s'en étaient approchées pour en examiner l'apparence.

Le premier arbre à pièces connu est un chêne déraciné sur l'Isle Maree, une petite île du Loch Maree dans les hautes terres du nord-ouest de l'Écosse. La première référence à de tels dépôts sur ce site provient de l'ouvrage de Thomas Pennant, A tour in Scotland and voyage to the Hebrides publié en 1775, dans lequel l'auteur décrit le puits sacré de l'île, qui aurait été consacré au viII siècle par saint Maelrubha - ou saint Maree -, et dont on racontait qu'il soignait la folie. Près du puits sacré se trouvait un arbre qui était utilisé comme «autel» : les pèlerins qui espéraient être guéris par le puits laissaient à saint Maelrubha un gage de leur gratitude, un ex-voto qu'ils déposaient sur cet arbre (Pennant 1775).

Ces gages semblent avoir été initialement des chiffons (bandes de tissu désignées sous le nom de clooties), qui étaient alors couramment utilisés comme offrandes votives ${ }^{2}$. Toutefois, au cours du xixe siècle, les pèlerins commencèrent à laisser d'autres objets, et notamment des clous et des épingles (Campbell 1860, Mitchell 1863). Ces objets métalliques, qui étaient à l'origine 
utilisés pour fixer les chiffons sur l'arbre, sont progressivement devenus des offrandes à part entière (Dixon 1886). Vers la fin du xixe siècle, ils semblent avoir été remplacés par des pièces.

En 1877, le site reçut une visiteuse célèbre: la reine Victoria, qui se rendit sur l'île Maree lors d'une tournée en Écosse. La reine et son escorte insérèrent eux-mêmes des pièces dans l'arbre, bien qu'à cette époque la pièce fût simplement considérée comme une offrande à saint Maelrubha plutôt que comme un soin contre la folie. Dans l'entrée de son journal datée du 17 septembre 1877, la reine Victoria décrit l'arbre en ces mots:

«On poussa le bateau sur la rive, et nous descendîmes à terre avant de traverser l'épais taillis de chênes, de gui et de hêtres qui recouvre toute l'île, jusqu'à parvenir au puits, aujourd'hui quasi asséché, dont on dit qu'il est connu pour guérir la folie. Un vieil arbre se trouve près du puits, et dans son tronc,

1. Arbre à pièces à Isle Maree, hautes terres du nord-ouest de l'Écosse depuis des temps immémoriaux, il est de coutume pour tous ceux qui se rendent en ce lieu d'insérer au moyen d'un marteau une pièce de cuivre, en guise d'offrande au saint qui vivait là au viII siècle... Nous insérâmes quelques pennies dans l’arbre à l'aide d'un marteau.» (Cité par Duff 1968.)

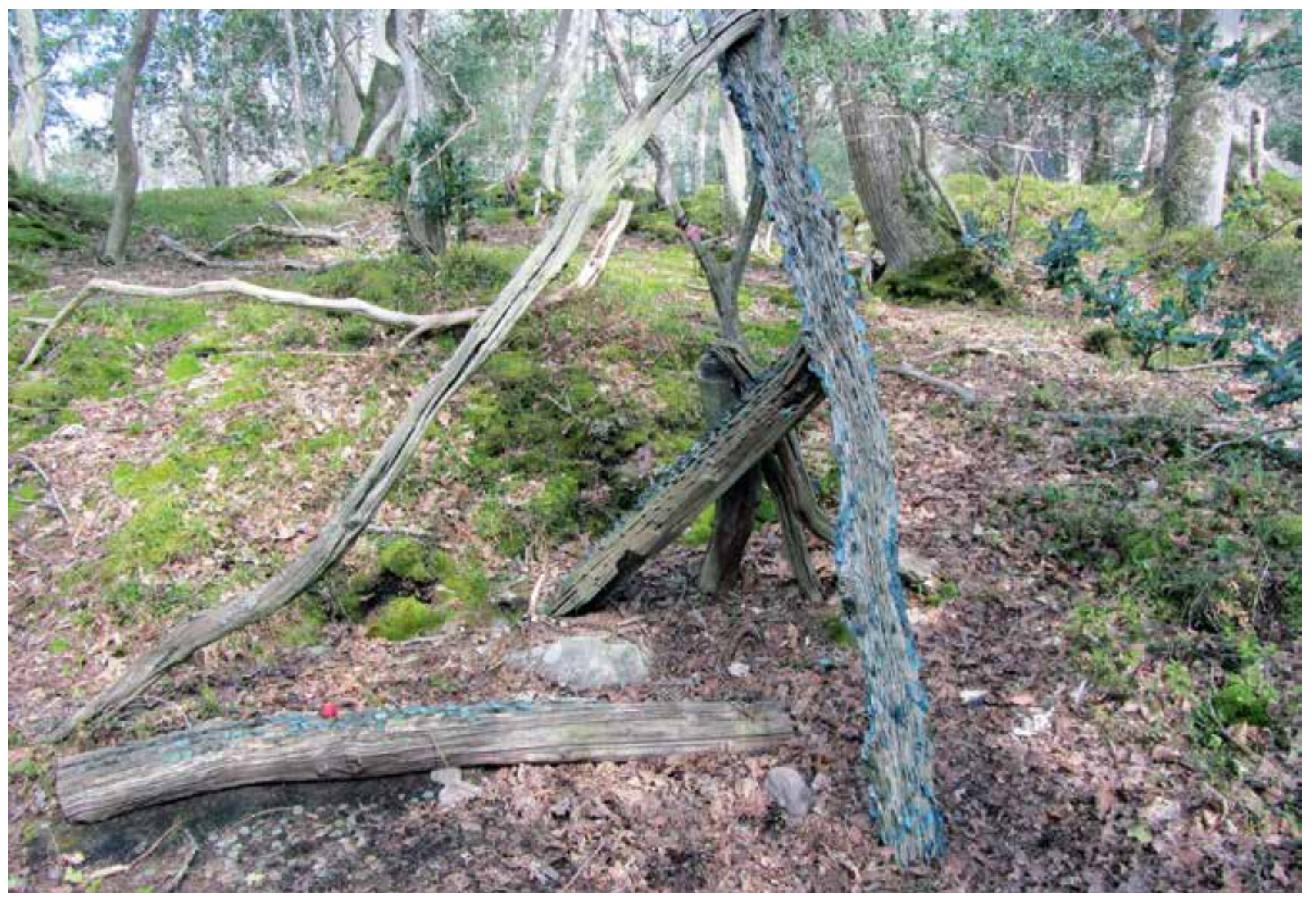


Il existe d'autres sites d'arbres à pièces en Écosse et en Irlande, dont l'histoire est tout aussi ancienne: Ardmaddy, dans le comté d'Argyll (Rodger et al. 2003), Clonenagh, dans le comté de Laois (Roe 1939, Morton 2004, Harbison 1991, Simon 2000), et Ardboe, dans le comté de Tyrone (Grimes 1999). Des arbres à pièces sont présents en ces lieux depuis la fin du XIXe et le début du $\mathrm{xx}^{\mathrm{e}}$ siècle. Toutefois, la majorité des 40 arbres à pièces catalogués à ce jour sont d'origine plus récente. Ils se trouvent le plus souvent dans des endroits publics gérés par des organismes de conservation et de protection de la nature comme le National Trust et la Forestry Commission, bien que ces organisations ne se considèrent pas comme étant à l'origine de la résurgence de cette coutume. Il semblerait que les pièces soient apparues spontanément dans les années 1990, 2000 et 2010 sous l'effet d'une conjonction fortuite de facteurs: la popularisation de la marche récréative, les nouvelles pratiques de gestion forestière consistant à laisser en place les arbres tombés plutôt que de les supprimer, ainsi que la diffusion via Internet de la connaissance de cette coutume ${ }^{3}$. Alors que la manifestation physique de la pratique consistant

Arbre à pièces sur le domaine d'Ardmaddy Estate, Argyll, Écosse

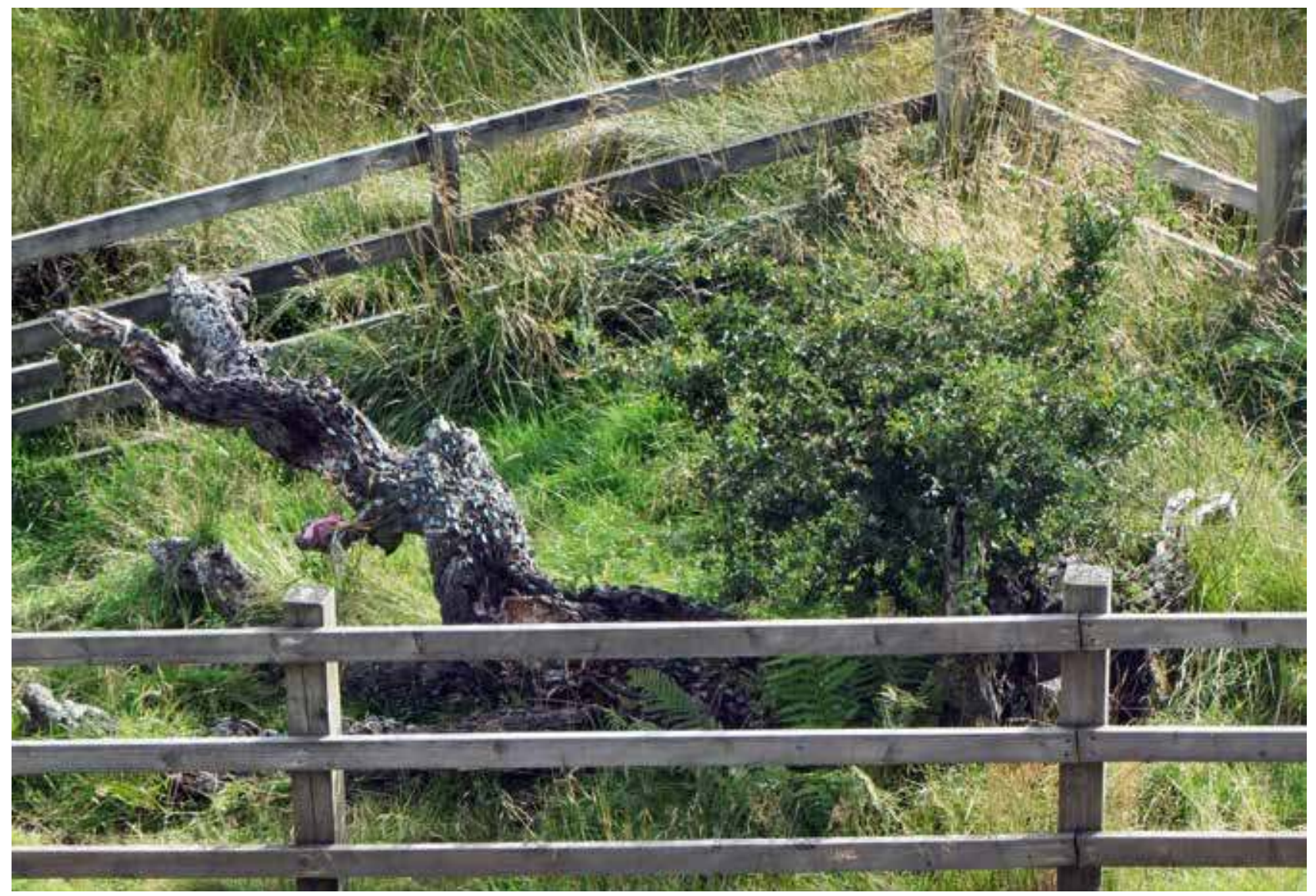


à incruster des pièces dans des arbres semble être restée la même entre les sites actuels et les occurrences plus anciennes en Écosse et en Irlande, sa «signification » a évolué.

Ainsi, à l'origine, les pièces étaient insérées dans certains arbres d'Isle Maree, de Clonenegh et d'Ardboe en guise d'ex-voto aux saints patrons du lieu, dans le but de guérir. Or, parmi les 200 personnes déposantes que j'ai pu interroger à propos de cette pratique entre 2011 et 2013, aucune n'a fait allusion à l'arbre à pièces comme vecteur éventuel de la guérison ou aux saints comme destinataires de ces pièces. Ce constat n'est guère surprenant dans la mesure où la maladie et la mort prématurée représentaient par le passé une menace beaucoup plus forte qu'aujourd'hui. Quelqu'un qui, de nos jours, s'inquiète pour sa santé est plus susceptible de consulter un médecin que de participer à un rituel d'échange (Hamilton 1981, Vyse 1997). Du reste, aucune des personnes ayant déposé une pièce n'a, en évoquant sa participation, fait référence à un culte, quel qu'il soit.

Aujourd'hui, les personnes déposantes invoquent une variété de raisons très différentes du vœu de guérison pour expliquer leur geste. Nombre d'entre elles avouent qu'elles ne participent à cette coutume que parce que d'autres l'ont fait avant elles: elles sont motivées par le fait de s'inscrire dans une «tradition». D'autres en appellent à l'esthétique comme principal facteur de motivation: elles voient l'arbre à pièces comme une œuvre d'art collective à laquelle elles souhaitent contribuer - une manière de «laisser sa marque », comme l'expliquent de nombreux participants. Ainsi, une femme déclare que déposer une pièce revient à «laisser un bout de soi qui sera vu par les autres ».

Toutefois, de nombreux autres participants - et en particulier les personnes accompagnées d'enfants - expriment l'idée que leur pièce a été laissée à l'arbre en échange de retombées positives: chance, fortune ou exaucement d'un vœu. Plusieurs personnes désignent l'arbre à pièces comme un «arbre à souhaits» ou «arbre porte-bonheur», par analogie avec le puits à souhaits, tandis que beaucoup d'autres font référence à l'idée de «pièce porte-bonheur ». Bien que ces données témoignent de la persistance d'une certaine intentionnalité, les pièces, pour ceux qui les déposent, n'ont plus valeur d'ex-voto. De surcroît, l'intention des participants, lorsqu'ils en ont une, est souvent vague: peu d'entre eux sont capables d'expliquer en détail ce qu'ils pensent être la visée de cette coutume, et aucun n'énonce une raison bien identifiée à sa participation. J'ai montré ailleurs que cette ambiguité et ce caractère polyphonique manifestent une «mutabilité du sens »: la coutume de l'arbre à pièces peut être interprétée de différentes manières selon le moment, le lieu et la personne qui y participe (Houlbrook 2014).

\section{Ia pièce, un dépôt spontané}

La monnaie est utilisée comme objet de dépôt rituel depuis des temps anciens, dans des contextes préchrétiens comme chrétiens. L'idée de se défaire d'une pièce en guise de paiement pour l'exaucement d'un vœu est encore présente à l'heure actuelle. On trouve très fréquemment 
des puits et des fontaines à voux dans les parcs, les destinations touristiques et les centres commerciaux à travers toute l'Europe et même le monde: depuis la fameuse Fontaine de Trévi à Rome jusqu'aux sites plus obscurs que nous pouvons rencontrer par hasard dans notre vie de tous les jours. Cependant, malgré cette popularité, la pièce n’a pas été créée pour servir de dépôt ou comme objet votif: elle a été conçue pour un usage séculier dans la vie quotidienne. Elle a donc, pour reprendre les termes d'Osborne, été « "convertie" jusqu'à devenir un objet susceptible d'être employé dans le cadre d'une transaction avec des pouvoirs surnaturels» (Osborne 2004) ${ }^{4}$. Comment cette conversion s'opère-t-elle, et pourquoi la pièce est-elle considérée comme un objet adapté pour un tel échange?

L'insertion d'une pièce dans un arbre à pièces est rarement un rituel planifié. Les personnes qui le pratiquent aujourd'hui ne se rendent pas de manière délibérée sur le site d'un arbre à pièces: au contraire, elles rencontrent ces arbres par hasard, et choisissent le plus souvent de participer à cette pratique improvisée en imitant celles qui les y ont précédées (Houlbrook 2016). Seules $17 \%$ des personnes interrogées avaient déjà rencontré un arbre à pièces auparavant. Les autres n'avaient pas connaissance de cette coutume et n'avaient donc pas apporté d'objet préalablement choisi pour être déposé. Dans son étude des arbres à rubans qui remonte au XIXe siècle, Walhouse remarquait que les participants ne s'étaient pas toujours préparés lorsqu'ils arrivaient à l'arbre. Sous l'effet de la nécessité, ils choisissaient leur dépôt parmi «les objet banals se trouvant à portée de la main : cornes, os, touffe de fourrure, lambeaux ou autre » (Walhouse 1880: 104). Cependant, Walhouse écrit au xixe siècle, époque à laquelle il évoque des coutumes internationales et historiques, depuis la Chine jusqu'au « Nouveau Monde». Dans les contextes qu'il étudie, les « objets triviaux se trouvant à portée de main » pouvaient en effet être des cornes, des os ou des touffes de fourrure. Ce n'est toutefois pas le cas dans la GrandeBretagne d'aujourd'hui. Or quel objet est à la fois trivial (c'est-à-dire jetable) et toujours à portée de main à la fin du xxe et au début du xxi siècle? La pièce de monnaie.

Même si cette situation est actuellement en train d'évoluer avec le développement du paiement électronique, la plupart des gens ont habituellement des pièces sur eux, que celles-ci se trouvent dans un porte-monnaie ou dans leur poche. Si une personne souhaite participer à un rituel spontané qui nécessite le «sacrifice » d'un objet, la pièce est donc l'objet le plus commode à utiliser. De plus, sa valeur n'est pas très élevée. Sur les 165360 pièces répertoriées sur tous les sites d'arbres à pièces, nous n'avons trouvé aucune pièce de 5 livres sterling (le montant le plus élevé pour une pièce), une seule pièce de 2 livres et seulement 25 pièces d'une livre. $92 \%$ des pièces étaient d'un ou deux pence, dont la valeur économique est aujourd'hui très basse. Ces pièces sont plus ou moins considérées comme jetables (Wolman 2012), et de nombreux participants contemporains admettent que c'est la raison pour laquelle ils les ont sélectionnées pour être déposées sur l'arbre. Du fait de leur ubiquité et de leur caractère jetable, les pièces représentent l'objet idéal pour un dépôt spontané.

Cependant, certaines des pièces incrustées dans les arbres à pièces montrent des indices de préméditation. Certaines, par exemple, ont été physiquement altérées: c'est le cas d'une pièce de deux pence trouvée à Dovedale dans le Derbyshire, sur laquelle la lettre « R» a été écrite au 


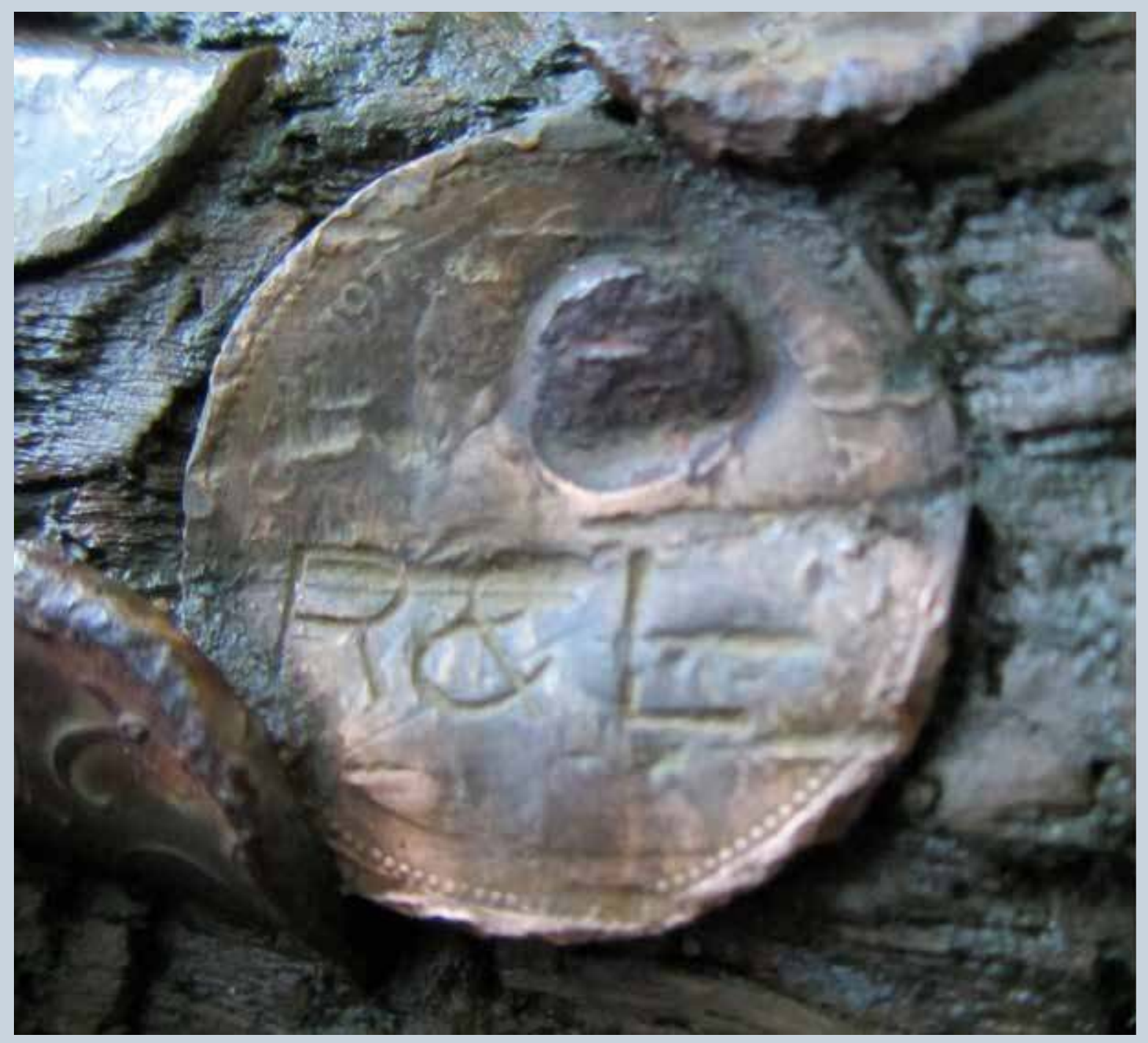

3. Pièce portant l'inscription « R \& $L$ » sur un arbre à pièces à Ingleton, Yorkshire, Angleterre 


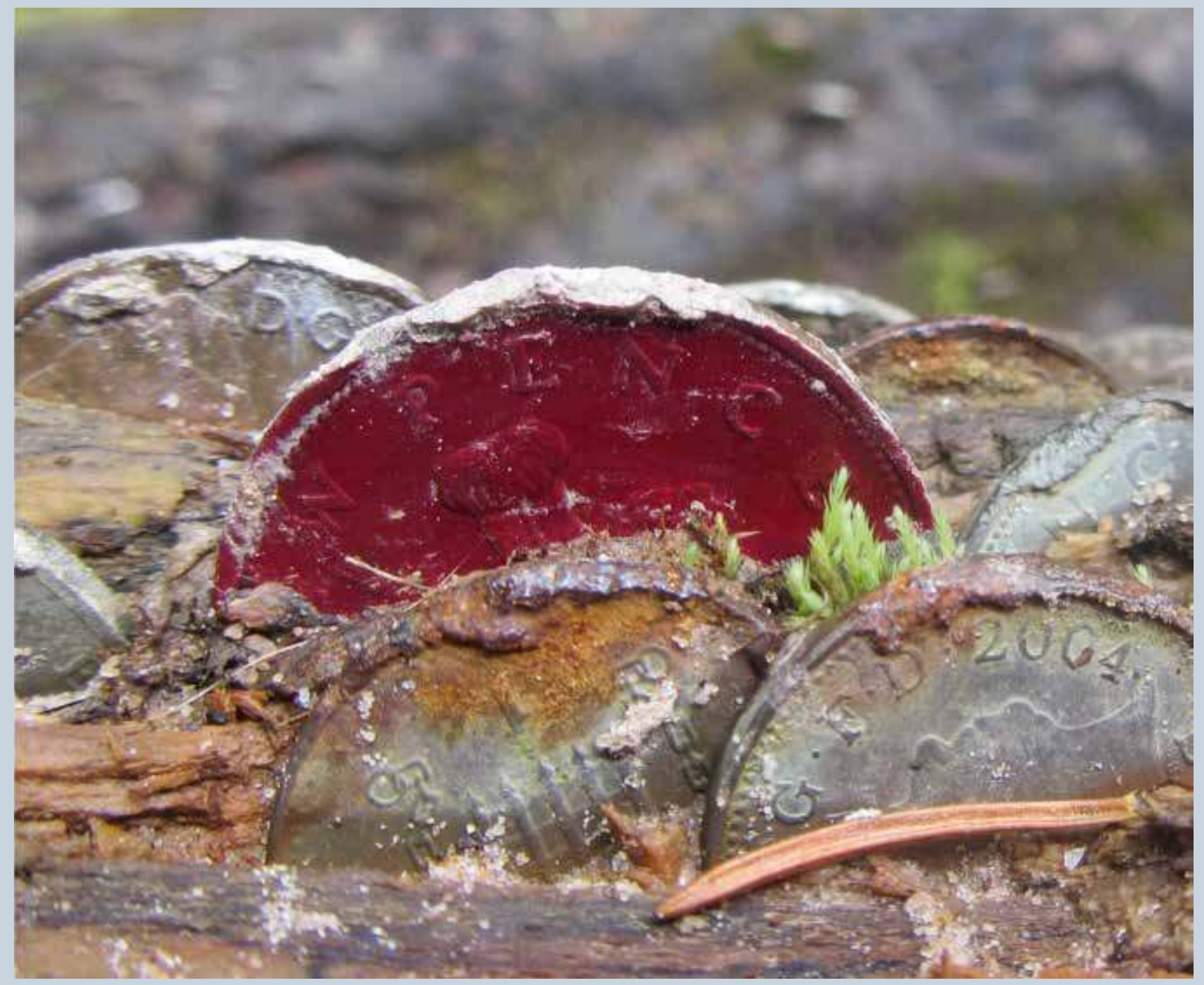

4. Pièce peinte en rouge dans un arbre à pièces à Fairy Glen, Black Isle, Écosse 
stylo argenté (on présume que le nom de la personne qui l'a déposée commençait par un « R»). De même à Ingleton dans le Yorkshire, les initiales « R \& L» ont été gravées sur une pièce de deux pence; tandis qu'à Fairy Glen sur l'île de Black Isle en Écosse, une pièce a été peinte en rouge, de toute évidence pour la distinguer des autres. À Ingleton également, sept pièces ont été clouées à l'arbre (parmi lesquelles celle sur laquelle figure « R \& L»), certainement pour fixer plus fermement les dépôts mais aussi pour les personnaliser. Cela indique de manière certaine que les personnes ayant déposé les pièces s'étaient munies de clous et peut-être même de marteaux, qui ne sont pas des objets que l'on transporte habituellement avec soi. Les pièces ne semblent donc pas toujours constituer un dépôt fortuit et improvisé.

5. Trois des sept pièces clouées à l'arbre à pièces d'Ingleton, Yorkshire

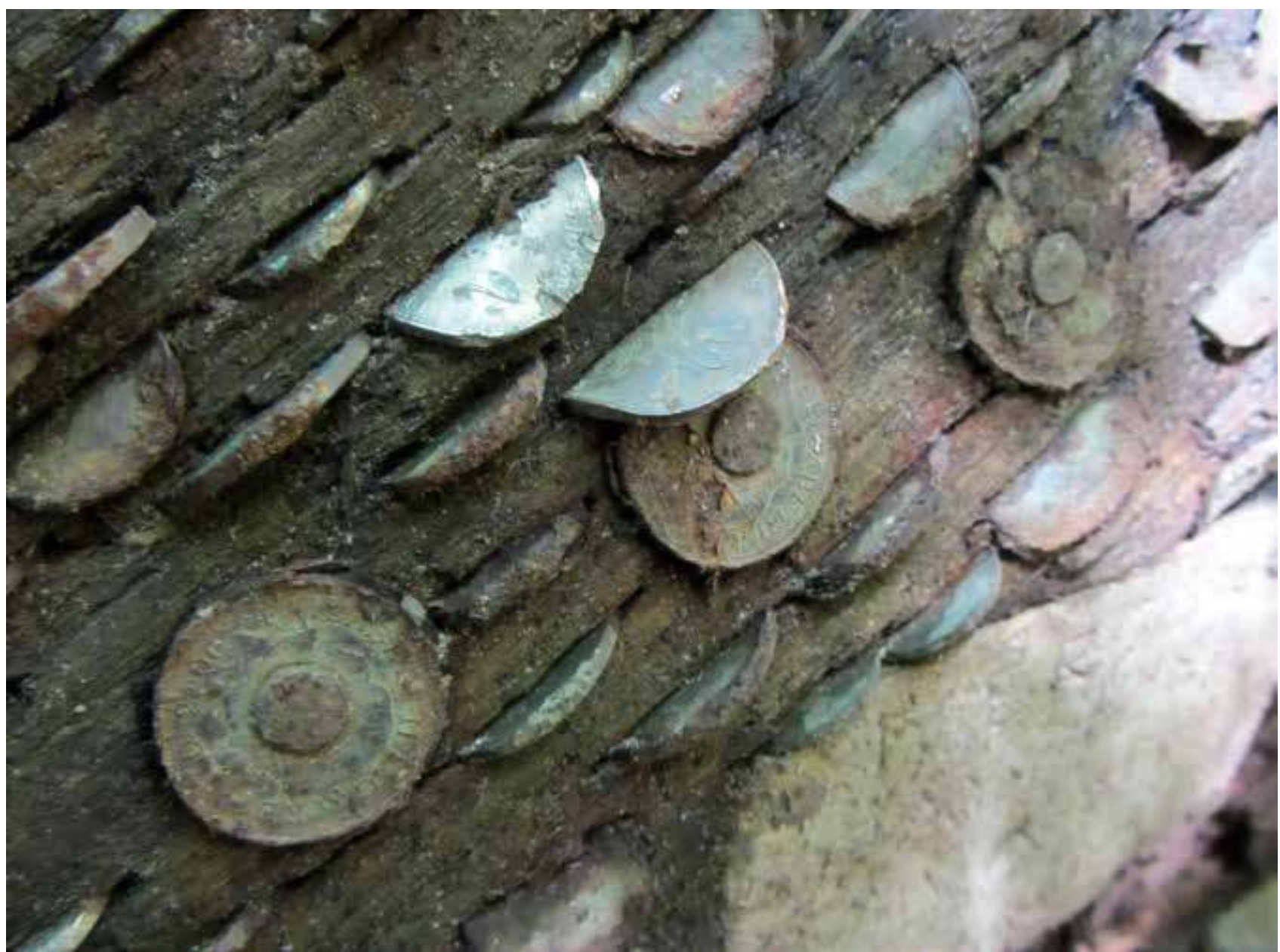




\section{Introductions aux cadenas d'amour}

Le cadenas d'amour est une autre forme de collaboration en série qui a proliféré au cours du $\mathrm{xxI}^{\mathrm{e}}$ siècle. La coutume consiste pour les couples à inscrire leurs noms ou leurs initiales sur un cadenas avant de l'attacher à une structure publique, le plus souvent un pont, comme le Ponte Milvio à Rome ou le pont de Brooklyn. Les clés sont ensuite jetées dans la rivière par le couple comme symbole de leur engagement mutuel. À Moscou, des structures métalliques en forme d'arbre ont été érigées spécialement à cet effet sur le pont Luzkhov, tandis qu'à Paris, sur le pont des Arts, l'accumulation de cadenas d'amour a atteint des proportions telles qu'une des balustrades du pont s'est écroulée sous leur poids en juin 2014. Bien que le cadenas d'amour soit déposé par un couple comme gage d'un vœu de rester ensemble plutôt que pour marquer la réalisation de ce vœu, celui-ci se rapproche de la notion d'ex-voto.

Les origines de cette pratique demeurent obscures, bien que des résidents de Vrnja ka Banja en Serbie affirment que leur agencement sur le Most Ljubavi (ou «Pont de l'Amour») remonte à la Première Guerre mondiale. Cependant, cette coutume a gagné en popularité suite à son adoption en Italie dans les années 2000, déclenchée par le roman d'amour de Federico Moccia, Ho voglia di te (J'ai envie de toi), paru en 2006, dans lequel un personnage accroche un cadenas au Ponte Milvio. Cette pratique se diffuse ensuite rapidement sans limites géographiques: des accumulations de cadenas d'amour apparaissent dans des lieux aussi divers et éloignés que New York, Séoul, Paris, Taïwan, Melbourne et Moscou.

Compte tenu de l'étendue de cette diffusion, il n'est guère étonnant que les cadenas d'amour aient déjà été examinés par la recherche académique sous des perspectives variées. L'historienne de l'art Cynthia Hammond (2010) étudie un agencement à Pécs en Hongrie qui remonte aux années 1980, et montre comment celui-ci peut être vu comme figurant le contrôle et la dissidence dans la ville. Jekaterina Lavrinec, scénographe urbaine (2013) catégorise cette coutume comme un «rituel urbain» en considérant les expériences corporelles qui entourent le dépôt du cadenas d'amour. L'ingénieur Christian Walloth (2014) décrit les cadenas d'amour comme dotés d'« un caractère émergent [c'est-à-dire non-prémédité et imprévisible] », et examine leur influence sur le développement urbain. L'artiste Lachlan MacDowell considère les cadenas d'amour dans le contexte du street art en examinant leur dépôt à travers le concept de stigmergie, selon lequel «des pratiques urbaines s'accumulent dans l'espace, sans coordination directe » (2015 : 41). Enfin, le sociologue Kai-Olaf Maiwald (2016) adopte une approche objective et herméneutique dans son enquête sur la signification symbolique du «padlocking (dépôt de cadenas) sur le pont Hohenzollern à Cologne.

J'ai pour ma part ajouté à ce corpus une approche centrée sur la culture matérielle, en récoltant des données sur 100 agencements de cadenas d'amour à travers le monde, et en répertoriant les 700 cadenas d'amour décrochés en 2016 du Centenary Bridge à Leeds (Royaume-Uni). Par ailleurs, à partir d'une enquête localisée sur la croissance d'un agencement de cadenas d'amour sur un pont de Manchester au Royaume-Uni entre février 2014 et aujourd'hui, j'ai pu 


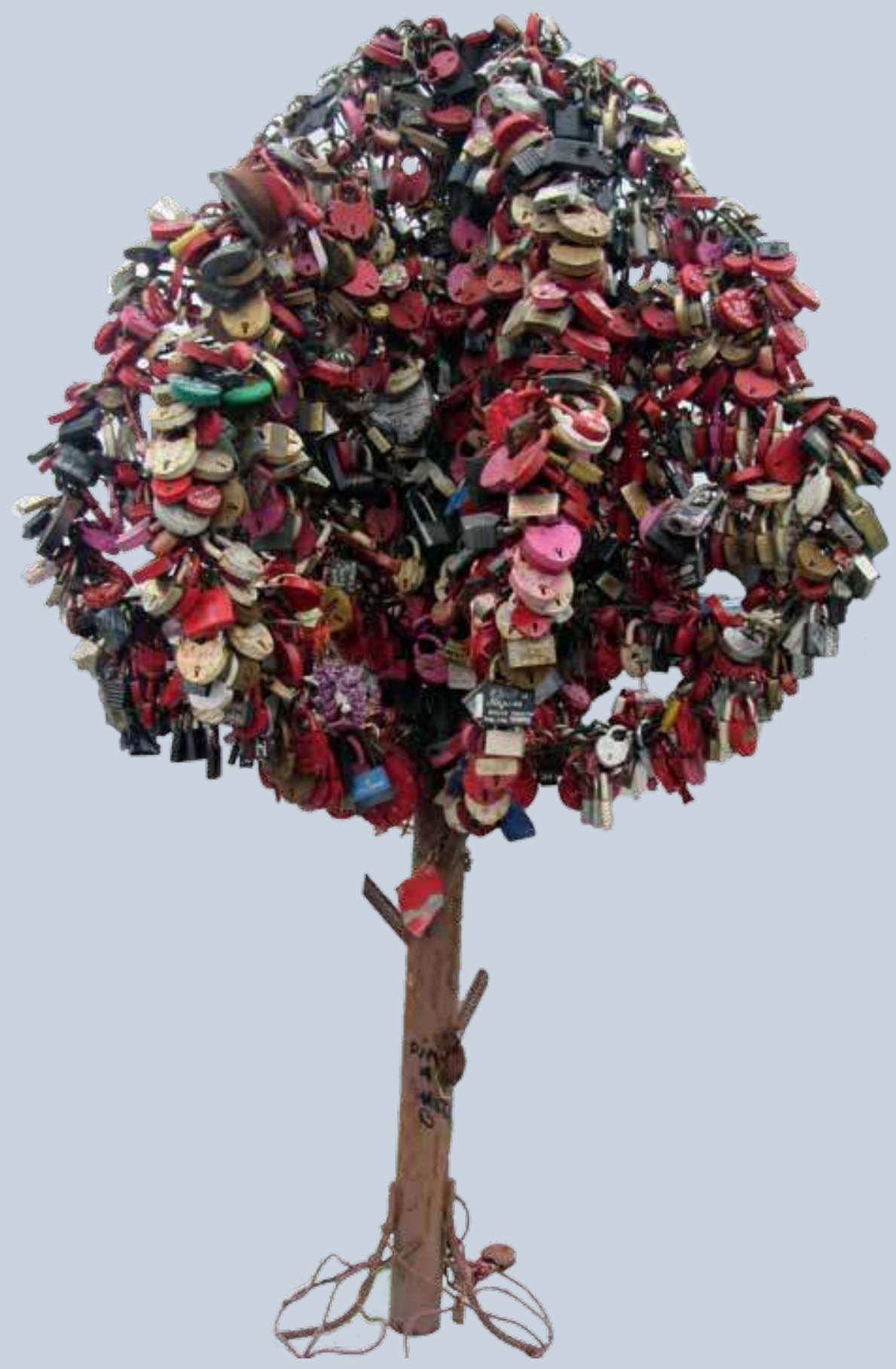


démontrer l'intérêt que présente l'étude d'un agencement sur la durée de sa formation plutôt qu'à un moment fixe dans le temps (Houlbrook 2018c). Que nous apprennent ces enquêtes sur le cadenas d'amour en tant qu'objet d'un dépôt prémédité?

\section{Le cadenas d'amour, un dépôt prémédité}

Bien qu'il soit vrai que c'est le plus souvent par hasard que l'on passe à côté d'un assemblage de cadenas d'amour, la décision de participer à cette coutume ne saurait être spontanée. À la différence d'une pièce de monnaie, il est rare que l'on ait un verrou sur soi : il ne s'agit pas d'un objet que l'on garde dans sa poche. Les cadenas ne répondent pas à la définition énoncée par Walhouse d'un « objet trivial se trouvant à portée de main ». S'il n'est généralement pas difficile de trouver un cadenas à acheter - surtout dans un environnement urbain -, cet achat nécessite que l'on quitte le site de l'assemblage pour trouver une boutique adaptée, puis que l'on retourne sur le site. On ne peut pas parler ici de dépôt improvisé car le cadenas n'est pas en général un objet commode à déposer, contrairement à une pièce de monnaie.

On a vu que les pièces sont sélectionnées comme objet de dépôt parce qu'elles n'ont pas une valeur particulièrement élevée. On peut en dire autant des cadenas: il est possible d'en acheter dans les magasins de la chaîne de bricolage Wilco pour 1,95£ (environ 2,20€). Du fait de leur faible valeur économique, ceux-ci pourraient donc être catégorisés parmi les dépôts improvisés. Cependant, il s'agit rarement de cadenas ordinaires. Certains d'entre eux, comme les cadenas magnifiquement ouvragés qui ornent le Centenary Bridge à Leeds ou Erzsébet Square à Budapest, ou bien les cadenas géants du pont Luzhkov à Moscou ou de Prague, ont visiblement de la valeur. D’autres ont été gravés par un professionnel pour y inscrire des noms, des messages ou même des images. Souvent, ces cadenas ont été commandés sur un des nombreux sites Internet qui font de la publicité pour leurs services de gravure, comme Make Love Locks, Beloved Padlocks ou Lock-itz. On peut ainsi commander un cadenas en forme de cœur gravé pour 19,99£ sur Love Locks UK, un site qui affiche le message suivant: «Du fait d'une demande élevée, le délai d'envoi des commandes est actuellement de 7 à 14 jours $»^{5}$. Compte tenu du prix et du délai, le cadenas gravé n'est de toute évidence pas assimilable à un dépôt improvisé.

Même les cadenas plus simples et bon marché ont en grande majorité été personnalisés. Tandis que certains portent simplement une inscription au marqueur (noms, initiales, dates, messages, assez comparables aux graffitis et aux noms gravés sur les arbres), certains ont été embellis à grand renfort de créativité. Le corps des cadenas est parfois couvert de dessins, depuis le bonhomme en bâtons jusqu'au bourdon, ou bien décoré d'autocollants. L'anse est parfois ornée de rubans ou recouverte de bande adhésive fantaisie. Certains ont été peints avec du vernis à ongles et des paillettes, d'autres recouverts de rosettes en tissu ou de petits habits tricotés à la main. De toute évidence, ces cadenas ont été habillés avant que le participant ne se rende sur le site: il s'agit d'actions organisées, préméditées, qui indiquent que certaines personnes rendent

6. Un arbre à cadenas sur le pont Luzhkov, Moscou 


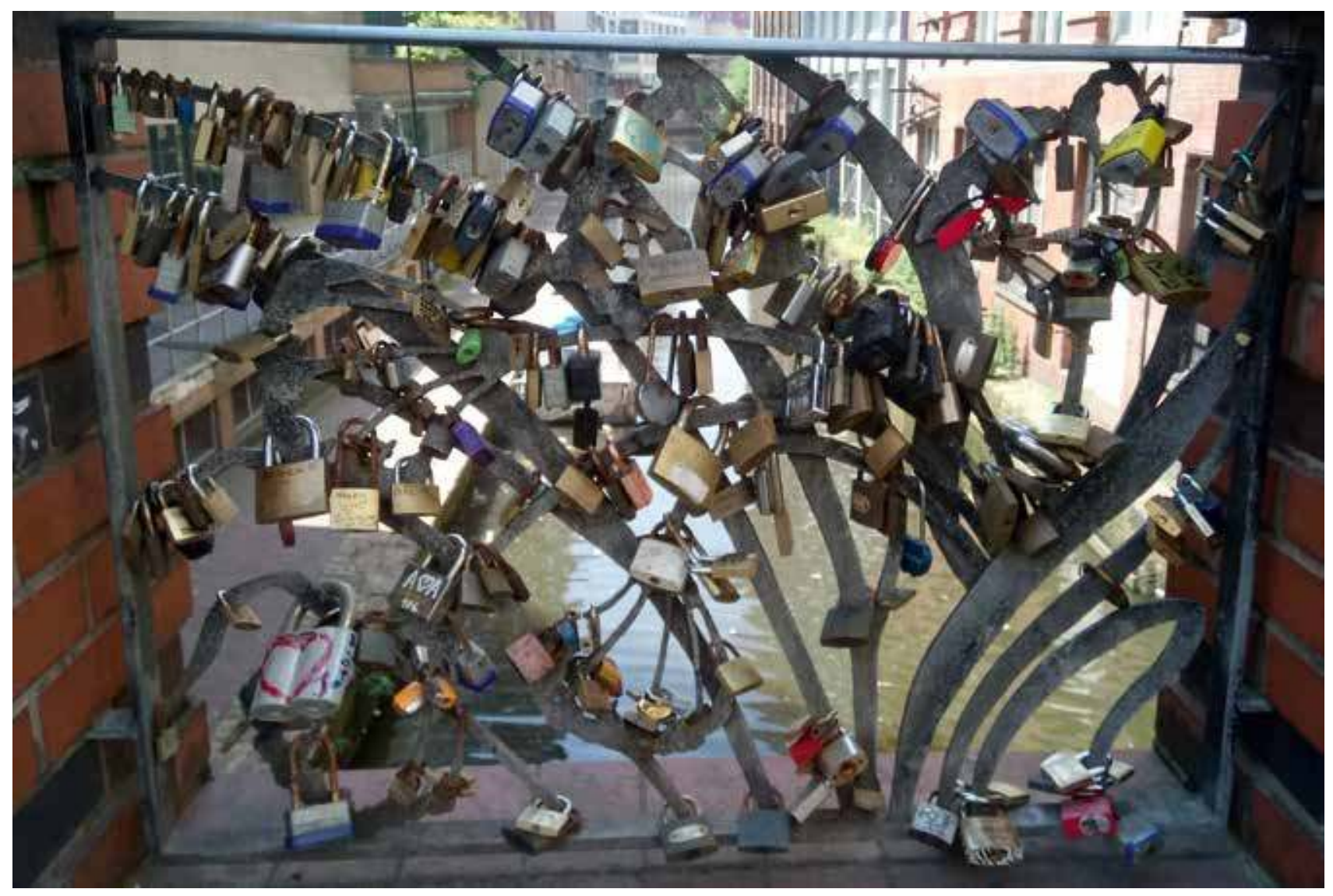

7. Un panneau du pont des cadenas d'amour à Manchester, Angleterre visite à ces assemblages de manière intentionnelle. Le nombre de dates de mariages et d'anniversaires de mariage inscrites sur les cadenas d'amour tend à indiquer qu'un couple choisit le moment juste pour déposer un cadenas - à la différence du dépôt de la pièce dans l'arbre à pièces, pour lequel le moment approprié est simplement celui où l'on rencontre un tel arbre.

On trouve cependant quelques exceptions à cette conception du cadenas d'amour comme dépôt prémédité. L'une d'elles est le Pont Neuf à Paris, où des marchands ambulants sont installés sur toute la longueur du pont pour vendre des cadenas gravés d'une image de la Tour Eiffel (certains n'ont pas même de clé, ce qui montre que les cadenas n'ont qu'une seule fonction). Cet assemblage de cadenas d'amour attire invariablement les touristes, à qui les marchands proposent leurs produits. Un marchandage s'ensuit et, une fois que le touriste a fait son achat, le colporteur lui propose un feutre marqueur pour qu'il puisse écrire son message sur le cadenas. De même à Vérone, la boutique de souvenirs de la «Maison des Capulet» - où des cadenas sont régulièrement accrochés - vend toutes sortes de cadenas en forme de cœur qui sont présentés comme des «Cadenas d'Amour Officiels»; pour plus de commodité, ceux-ci sont vendus avec un feutre 
marqueur noir. Dans ces derniers cas, les cadenas d'amour peuvent donc être considérés comme des dépôts spontanés. Les participants peuvent prendre une décision improvisée sur place et prendre part à la coutume, comme c'est le cas pour les arbres à pièces.

Les agencements populaires contemporains sont souvent spontanés et imprévus, depuis les cairns de montagne jusqu'aux puits à souhaits. Ce caractère se manifeste par la nature même des objets déposés, qui sont généralement de faible valeur économique et facilement disponibles lorsqu’une personne rencontre par hasard un agencement. Cela indique une certaine spontanéité et, peut-être par extension, une absence d'intentionnalité. La pièce dans un arbre à pièces est un exemple typique d'ex-voto qui a, au cours du temps, perdu toute véritable intentionnalité. Toutefois, le cadenas d'amour prouve que les dépôts contemporains ne sont pas toujours spontanés. Achetés, commandés ou décorés à grand renfort de créativité avant la visite à l'assemblage de cadenas d'amour, bon nombre de ces cadenas sont de toute évidence déposés de manière préméditée. Il en découle une plus

8. Cadenas d'amour décorés sur la place Erzsébet, Budapest, Hongrie grande intentionnalité et un investissement émotionnel plus grand dans le rituel (à défaut de croyance).

De manière générale, la dichotomie entre ces pratiques paraît évidente. Les pièces sont le plus souvent l'objet de dépôts spontanés, tandis que les verrous d'amour sont déposés de manière préméditée. Cependant, il ne s'agit pas d'une règle immuable: certaines pièces, modifiées et décorées avant d'être incrustées dans le tronc de l'arbre, indiquent une préméditation. À l'inverse, certains cadenas, achetés sur place avec un feutre marqueur, reflètent une décision spontanée de participer. Ces exemples suggèrent donc moins une dichotomie entre dépôt «spontané» ou «prémédité » qu'une continuité entre ces deux pôles. Les dépôts contemporains mettent ainsi en évidence un continuum d'intentionnalité et d'investissement qui mériterait d'être examiné plus en profondeur. On pourrait notamment étudier plus en détail les pratiques de dépôt rituel en lien avec le tourisme: si les pièces dans les arbres à pièces comme les cadenas d'amour s'apparentent à l'ex-voto, ceux-ci s'en distinguent par les raisons pour lesquelles des individus - principalement des touristes - participent aujourd'hui à ces coutumes. Une étude détaillée de ces ressorts permettrait de mieux comprendre la manière dont les pratiques votives ont dû s'adapter pour survivre.

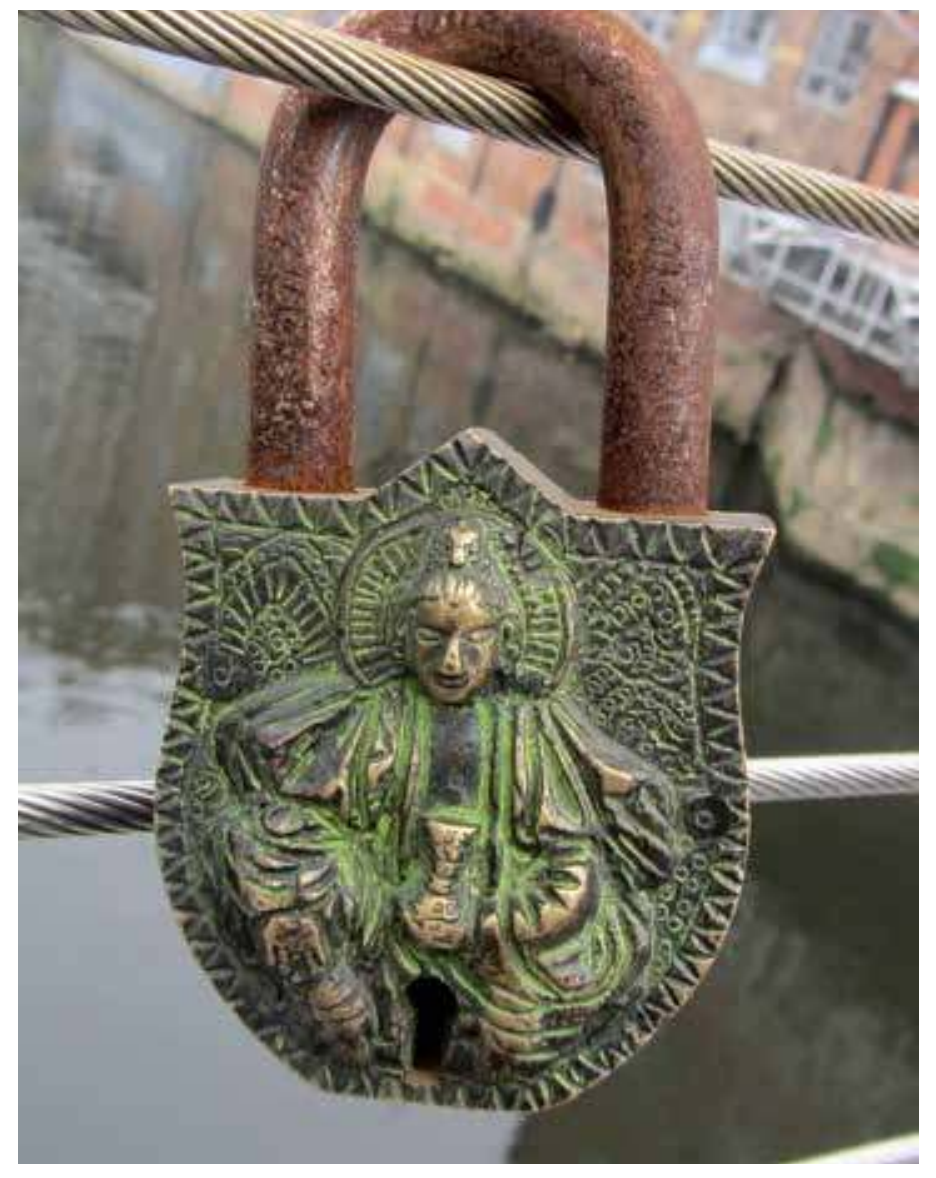




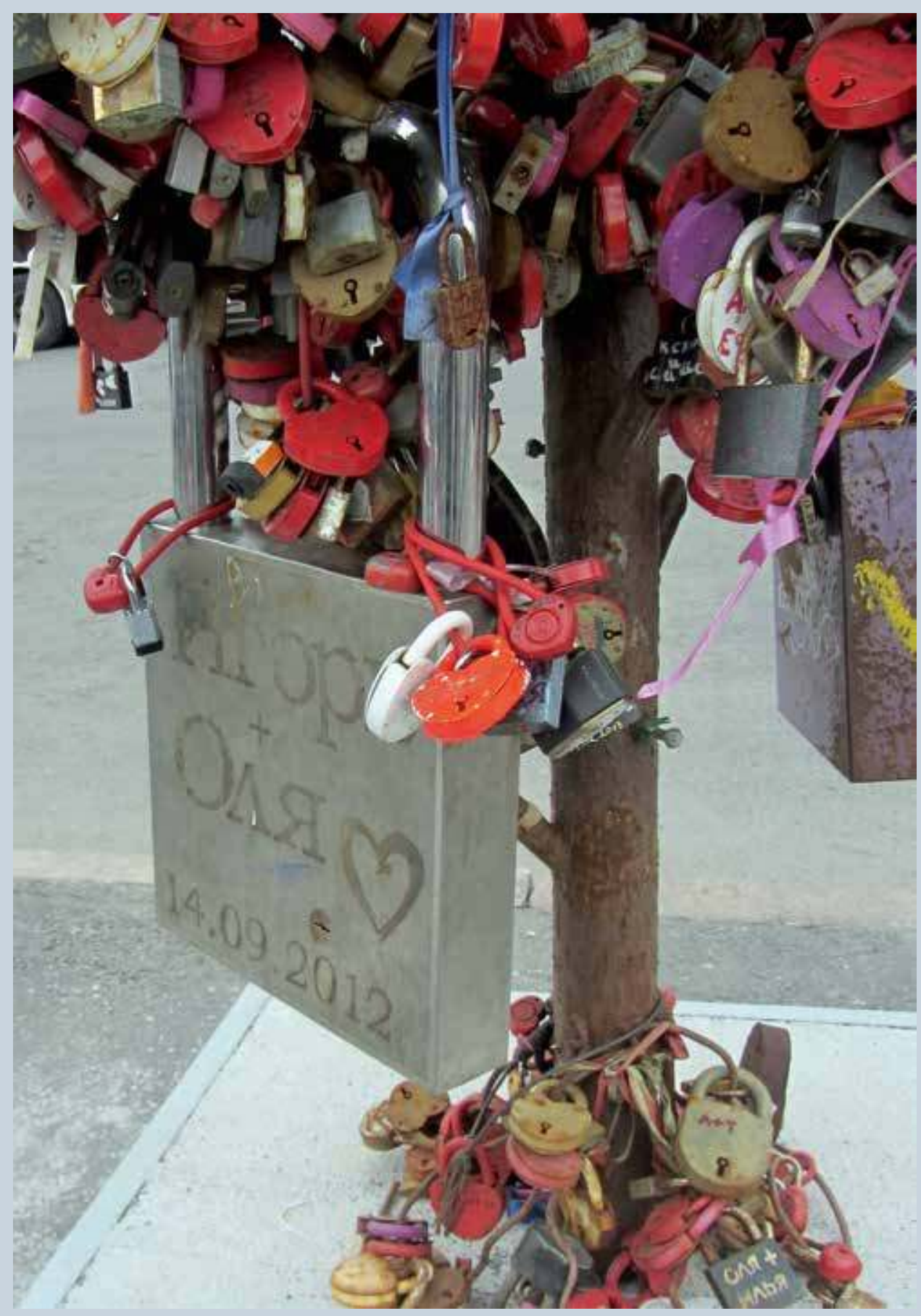

9. Cadenas d'amour géants sur le pont Luzhkov, Moscou 


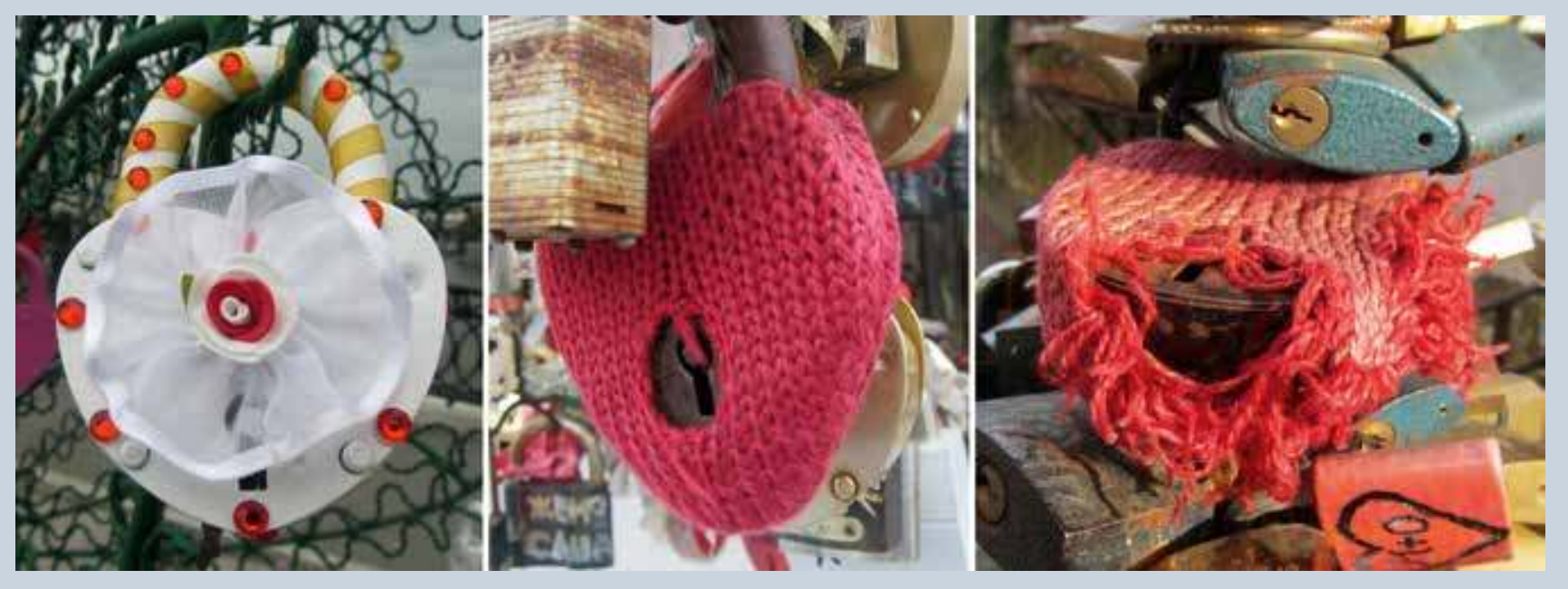

10. Cadenas d'amour décorés à Moscou et à Prague

11. Cadenas d'amour vendus avec un feutre marqueur dans la boutique de souvenirs de la «Maison des

Capulet » à Vérone

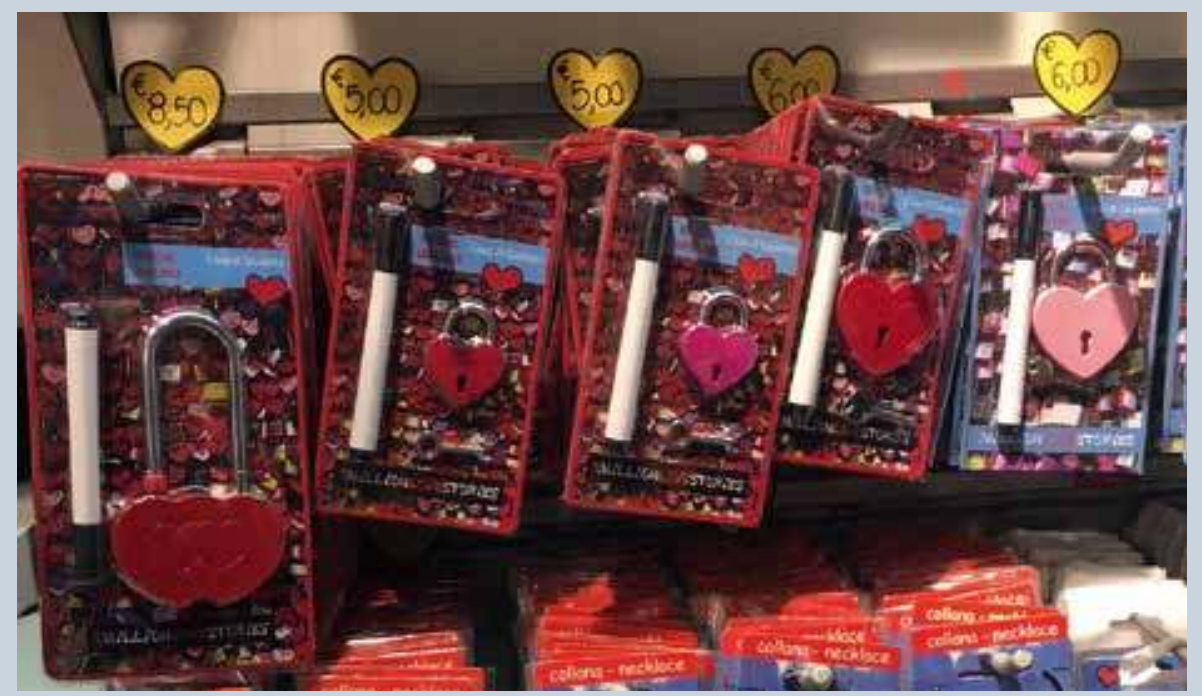




\section{Notes}

1. Pour un examen plus approfondi de cette pratique, voir Houlbrook 2014, 2016, 2017, 2018a, 2018b.

2. Les chiffons et vêtements ont longtemps été utilisés comme dépôts votifs dans les îles britanniques, le plus souvent accrochés aux branches d'arbres ou de buissons à proximité d'un puits sacré. Pour des études de ces sites et des approches théoriques concernant l'utilisation des chiffons comme vecteurs de transfert par contagion, voir Hardy (1840), Walhouse (1880), Hartland (1893), Hope (1893), Jones (1954), Lucas (1963), Bord \& Bord (1985),

\section{Iconographie}

Image d'ouverture. Arbre à pièces à Bolton Abbey, Yorkshire, Angleterre. Photographie: C. Houlbrook.
Shephard (1994), Rattue (1995), Healy (2001) et Rackard et al. (2001).

3. Pour une étude plus détaillée de ce phénomène, voir Houlbrook 2018.

4. J'étudie ce processus de manière plus approfondie dans Houlbrook 2015, 2016.

5. https://www.lovelocksuk.com/romance.html; https://www.makelovelocks.com/; http://www. belovedpadlocks.com.au/engraving-text-ideas/; http://lock-itz.com/

1 à 10. Photographies: C. Houlbrook.

11. Avec l'aimable autorisation de M. Gaskill.

\section{I'auteure}

Dr Ceri Houlbrook est chercheure en folklore et histoire à l'université de Hertfordshire. Ses principaux travaux portent sur le folklore contemporain et la culture matérielle des pratiques rituelles. Elle a coédité The Materiality of Magic, a publié The Magic of Coin-Trees from Religion to Recreation et prépare actuellement un ouvrage sur le phénomène mondial des cadenas d'amour.

\section{Références}

Bord, J. \& C. Bord 1985 Sacred Wells: Holy Wells and Water Lore in Britain and Ireland. London: Paladin Books.

Campbell, J. F. 1860 Popular Tales of the West Highlands. Vol. 2. Edinburgh: Edmonston and Douglas.

Dixon, J. H. [1886] 1984 Gairlock in North-West Ross-shire: Its records, Traditions, Inhabitants, and Natural History; with a Guide to Gairlock and Loch Maree and a Map of Illustrations. (4th reprint). Fort William: Nevisprint Ltd.

Duff, D. dir. 1968 Victoria in the Highlands. The Personal Journal of Her Majesty Queen Victoria: with Notes and Introductions, and a Description of the Acquisition and Rebuilding of Balmoral Castle. London: Frederick Muller Ltd.

Grimes, P. 1999 «The Pin Tree», Loughshore News (March)

Hamilton, D. 1981 The Healers: A history of Medicine in Scotland. Edinburgh: Canongate.
Hammond, C. 2010 «Renegade ornament and the image of the post-socialist city. The Pécs "love locks", Hungary» in T. Kovacs dir. The Present of the Recent Past: Reinterpretations of Socialist Modernism in East Central Europe. Vienna: Böhlau Verlag: 181-195.

Harbison, P. 1991 Pilgrimage in Ireland: The Monuments and the People. Syracuse: Syracuse University Press.

Hartland, E. S. 1893 «Pin-wells and Rag-bushes», Folklore 4 (4): 451-470

Healy, E. 2001 In Search of Ireland's Holy Wells. Dublin: Wolfhound Press Ltd.

Hope, R. C. 1893 The Legendary Lore of the Holy Wells of England. Including Rivers, Lakes, Fountains, and Springs. London: Elliot Stock.

Houlbrook, C. 2014 « The mutability of meaning. Contextualising the Cumbrian coin-tree», Folklore 125 (1): 40-59. 
- 2015 «Small change. Economics and the British cointree», Post-Medieval Archaeology 4 (1) : 114-130.

- 2016 "Because other people have done it". Cointrees and the aesthetics of imitation», Journal of Contemporary Archaeology 2 (2) : 243-261.

— 2018a The Roots of a Ritual. The Magic of Coin-trees from Religion to Recreation. Cham: Palgrave Macmillan. DOI: 10.1007/978-3-319-75517-5.

- 2018b «Why money does grow on trees. The British coin-tree custom», in M. N. Burström \& G. Ingvardson dir. Divina Moneta. Coins in Religion and Ritual. Routledge: Oxon and New York: 87-107.

- 2018c «Lessons from love-locks. The archaeology of the contemporary assemblage », Journal of Material Culture 23 (2) : 214-238.

Hulse, T. G. 1995 «A modern votive deposit at a North Welsh holy well», Folklore 106: 31-42.

Jones, F. 1954 The Holy Wells of Wales. Cardiff: University of Wales Press.

Lavrinec, J. 2013 «Urban scenography. Emotional and bodily experience», Limes: Borderland Studies 6 (1): 21-31.

Lucas, A. T. 1963 « Sacred Trees of Ireland », Journal of the Cork Historical and Archaeological Society 68: 16-54.

MacDowall, L. 2015 «Graffiti, street art and theories of stigmergy in J. Lossau \& Q. Stevens dir. The Uses of Art in Public Space. London: Routledge: 33-48.

Maiwald, K. 2016 «An ever-fixed mark? On the symbolic coping with the fragility of partner relationships by means of padlocking», Forum: Qualitative Social Research 17 (2).

McNeill, L. 2007 «Portable places. Serial collaboration and the creation of a new sense of place», Western Folklore 66 (3/4) : 281-299.

Mitchell, A. 1863 «On various superstitions in the NorthWest Highlands and islands of Scotland, especially in relation to lunacy », Proceedings of the Society of Antiquaries of Scotland 3: 251-288.

Morton, A. 2004 Tree Heritage of Britain and Ireland. Marlborough: Airlife Publishing.
Osborne, R. 2004 «Hoards, votives, offerings. The archaeology of the dedicated object », World Archaeology 36 (1): $1-10$.

Pennant, T. 1775 A Tour in Scotland and Voyage to the Hebrides, MDCCLXXII. Vol. 2. Dublin: A. Leathley.

Preston, C. 2007 «Panty trees, shoe trees, and legend», FOAFTALE News: News Letter of the International Society for Contemporary Legend Research 67 (May): 11-12.

Rackard, A., O'Callaghan, L. \& D. Joyce 2001 Fish Stone Water: Holy Wells of Ireland. Cork: Cork University Press.

Rattue, J. 1995 The Living Stream: Holy Wells in Historical Context. Woodbridge: Boydell Press.

Rodger, D., Stokes, J. \& J. Ogilvie 2003 Heritage Trees of Scotland. London: Tree Council.

Roe, H. M. 1939 «Tales, customs and beliefs from Laoighis», Béaloideas 9 (1): 21-35.

Santino, J. 2004 «Performative commemoratives, the personal, and the public. Spontaneous shrines, emergent ritual, and the field of folklore», Journal of American Folklore 117: 363-372.

Shephard, V. 1994 Historic Wells In and Around Bradford. Loughborough: Heart of Albion Press.

Simon, B. 2000 «Tree traditions and folklore from Northeast Ireland », Arboricultural Journal 24 (1): $15-40$.

Vyse, S. A. 1997 Believing in Magic: The Psychology of Superstition. Oxford and New York: Oxford University Press.

Walhouse, J. 1880 «Rag-bushes and kindred observances », The Journal of Anthropological Institute of Great Britain and Ireland 9: 97-106.

Walloth, C. 2014 «Emergence in complex urban systems. Blessing or curse of planning efforts? » in C. Walloth et al. dir. Understanding Complex Urban Systems: Multidisciplinary Approaches to Modeling. Berlin: Springer.

Wolman, D. 2012 The End of Money. Counterfeiters, Preachers, Techies, Dreamers and the Coming Cashless Society. Boston: Da Capo Press.

\section{Pour citer l'article}

Houlbrook, C. 2018 «Des pièces de monnaie au cadenas. Un spectre des dépôts contemporains », TechniquesE Culture 70 «Matérialiser les désirs. Techniques votives», p. 240-259. 